\title{
Risk analysis of production process for food SMEs using FMEA method: a case study
}

\author{
Prima Fithri $^{1^{*}}$, Muhammad Rafi $^{1}$, Pawenary ${ }^{2}$, and A. S. Prabuwono ${ }^{3}$ \\ ${ }^{1}$ Department of Industrial Engineering Faculty of Engineering, Andalas University, Indonesia \\ ${ }^{2}$ Electrical Engineering, Faculty of Engineering, ITPLN, Indonesia \\ ${ }^{3}$ Department of Information Technology, Faculty of Computing and Information Technology in Rabigh, King \\ Abdulaziz University, Saudi Arabia
}

\begin{abstract}
The increasing development of the industry makes every industry have to compete with other competitors to gain an edge. The advantages of competition are influenced by several factors, one of which is good human resource management. Where if a company has good human resources, it will increase profits indirectly and can increase productivity. This research discusses case studies about the potential dangers of IKM Heppy Bakery's potential dangers that can harm workers in bread production. The method used is Failure Mode And Effect Analysis (FMEA). Later, the data will be filled and given a rating distinguished into three parts: severity, occurrence, and detection. The data were obtained through questionnaires given to 3 workers at IKM Heppy Bakery and filled in rating values based on the provisions that have been given to the questionnaire. This Value helps determine the Risk Priority Number (RPN) obtained from multiplication between severity, occurrence, and detection. After processing the RPN multiplication data, the highest RPN value was obtained by 193 with the danger factor of the operator overheating and dehydrating due to high temperatures. Furthermore, the calculation of critical Value was obtained by 109. Based on the critical Value obtained seven hazard factors above the critical value, these seven hazard factors need to be improved so that workers do not avoid accidents when conducting the production process.
\end{abstract}

\section{Introduction}

Currently, the government makes Small and Medium Industries (IKM) a focus in development. Therefore, Small and Medium Industries are one of the pillars of the country's economy, which is strong enough to fight the world economy that can use many resources to create jobs for organizations. In addition, Small and Medium Industries need training or socialization, especially those related to the safety and health of workers. Most workers who work in Small and Medium Industries have low jobs and irregular working hours, and the wages given are not sufficient for workers' needs. Some hazards found in the workplace are high work environment temperatures, poor workplace lighting, inhaled dust, and exposure to chemicals used. A form of business that can increase its productivity by having good resources, the company must pay attention to the safety and health of the workers as well as possible.

The safety and health of workers is an obligation that the company must consider (BBC NEWS, 2010; Ishimaru et al., 2020). Occupational safety and health must be considered because it will cause work accidents that impact worker productivity [3]. Small and Medium
Industries (IKM) know the importance of Safety, Health, and Environment (SHE) in the business sector. They lack the SHE field that company owners must consider, which can be seen in IKM Happy Bakery. These SMEs pay less ${ }^{\dagger}$ attention to the application of SHE in the production process carried out by workers.

Potential hazards are often as operators who work often experience overheating and dehydration due to a narrow working space; operators are fatigued due to the position of items that are not ergonomic; operators do not wear protection when mixing mashing dough which can result in pinched hands. Based on the problems in the Happy Bakery IKM, the authors complete the case study to identify what risks can be encountered in the IKM during production. This is done so that IKM Heppy Bakery can apply the importance of Safety, Health, and Environment (SHE) in the work environment, increasing worker productivity and providing benefits for the company. This case study was completed by observing the potential work hazards in the production process to prevent work hazards arising from hazards during the production process at IKM Heppy Bakery.

The problem raised comes from a previous study that discusses determining priorities for overcoming work

\footnotetext{
*Corresponding author: primafithri@eng.unand.ac.id
} 
accidents using the FMEA method [4]. The result of this study is that 12 kinds of work accidents occur at the research site. In addition, a previous study was conducted regarding the dangers of the machines using the FMEA method [9]. This research shows that the body plate component is the main hazard that affects the engine most. Based on previous research conducted by Paciarotti $\mathrm{C}$ et al. (2014), which discussed the A revised FMEA application to the quality control management, the results obtained were cost savings time efficiency (Paciarotti et al., 2014). In addition, based on previous research conducted by Doshi, J., and Desai, D. which discussed the application of Failure Mode \& Effects Analysis (FMEA) For Continuous Quality Improvement Multiple Case Studies in Automobile, it was found that to identify potential failures that occurs in the production process and product quality can use the FMEA method [2].

From that previous study, this method can be used for this case study to know the potential priorities danger in this IKM.

\section{Research Methodology}

The stages of observing problem objects and methods used in case studies start from preliminary studies, literature studies, problem identification and formulation, data collection, data processing, analysis, and conclusions and suggestions.

A preliminary study is to identify the actual circumstances of the event at the company. This stage is a stage that conducts direct observations about the process of making bread at the IKM Heppy Bakery. This direct observation found a problem about the potential risk of harm to workers during the production process.

Literature studies are done hand in hand with field studies. This study is also helpful for solving problems found. In this literature study, all references related to field studies or problems will be resolved. References help formulate problems, data processing, and analysis. Some theories related to the problem are Risk, Risk Management, Damage, Maintenance, Failure Mode, and Effect Analysis (FMEA).

The procedure of problem identification is done by paying attention to the circumstances when processing bread production at IKM Heppy Bakery. The observations showed a risk that could potentially occur hazards when conducting the production process

The data was collected regarding potential hazards that can occur when workers during the production process. This potential hazard data is obtained from questionnaires filled by three factory workers. These three workers work on the grinding machine operator, dough mixer, and roasting, where they later fill out the questionnaire given. This data is needed to calculate the FMEA value and find the main Risk Priority Number (RPN) sequence that has the potential for hazards in doing the job.
After the data is collected, it will be done against the data. This research uses the FMEA method to analyze what risks are potentially dangerous when doing the production process. A rating does this FMEA method based on severity, occurrence, and detection rating. Furthermore, the RPN value is calculated to determine which priority is first improved. The formula used in calculating RPN Value.

$$
R P N=S \times O \times D
$$

Formula description:

$R P N=$ Risk Priority Number

$S=$ Severnity

$O=$ Occurance

$D=$ Detection

When you have obtained the RPN value, further calculation of critical Risk Priority Number (RPN) is used to see how many failure modes are above RPN critical Value and provide solutions to factors that risk the occurrence of hazards in work. The formula of the critical value is.

Critical Value $=\frac{\text { RPN Total }}{\text { Number of Risks }}$

The results of data processing are analyzed to evaluate the problems that have been solved. First, the analysis can be done by looking at the main potential that causes the hazard, then the sequence of potential hazards that can occur, then the critical value of the Risk Priority Number and suggestions for problem-solving against the risk of potential hazards exist.

\section{Results and Discussion}

The results and discussion obtained in this study are based on a data questionnaire given to 3 workers who do the process of bread production.

Respondent 1: Ana Laila (dough mixer machine operator) Respondent 2: Khairul Azmi (grinding machine operator) Respondent 3: Junior (Roasting Machine operator)

This questionnaire data contains rankings on factors that allow the occurrence of hazard risk when workers do the process of bread production in IKM Heppy Bakery is distinguished into three parts, namely Severity, Occurrence, and Detection that have been averaged can be seen in Table 1

After obtaining severity, occurrence, and detection values from 3 respondents, RPN calculation is done by multiplying three variables in the FMEA method that have been obtained and then done the process of sequencing RPN value to see the order of the highest causes of potential hazards in workers can be seen in Table 1. 
Table 1. RPN Scores from3 Respondents

\begin{tabular}{|c|l|c|c|c|c|}
\hline No. & \multicolumn{1}{|c|}{ Potential Hazards } & Severity & Occurance & Detection & RPN \\
\hline 1 & $\begin{array}{l}\text { Operator overheating and dehydration due to high } \\
\text { temperatures }\end{array}$ & 7 & 7 & 196 \\
\hline 2 & Narrow work areas cause accidents while working & 7 & 4 & 4 & 112 \\
\hline 3 & Hearing loss due to noise & 5 & 6 & 5 & 150 \\
\hline 4 & Improper body position as the operator fatigues & 6 & 5 & 5 & 150 \\
\hline 5 & Operator At Risk of burns in the grill & 5 & 4 & 5 & 100 \\
\hline 6 & The operator is concerned when using the seat as a tool & 5 & 5 & 6 & 150 \\
\hline 7 & Operator slips due to slippery floor & 6 & 5 & 4 & 120 \\
\hline 8 & May be electrocuted due to irregular cable position & 5 & 3 & 3 & 45 \\
\hline 9 & Inhaled dust causes disturbance to the smell & 3 & 6 & 5 & 90 \\
\hline 10 & $\begin{array}{l}\text { The operator's hand is at risk of getting pinched when } \\
\text { smoothing the dough }\end{array}$ & 4 & 2 & 6 & 48 \\
\hline 11 & Crash due to load stack overriding operator & 6 & 4 & 6 & 144 \\
\hline 12 & Potential gas leakage due to improper gas location & 7 & 2 & 2 & 28 \\
\hline
\end{tabular}

Here's an example of a calculation of the RPN value:

$$
\begin{aligned}
R P N \text { Scores } 1 & =S \times O \times D \\
& =7 \times 7 \times 4 \\
& =193
\end{aligned}
$$

Based on the sequence of RPN values of each factor that has the potential to cause harm to workers, further determining the critical Value of RPN to show what danger factors are above the critical value so that it needs to be evaluated improved quickly. Based on Table 1, as many as 12 factors are causing potential hazards with a total RPN value of 1313; based on the total value can be determined critical value RPN with the calculation using the formula:

$$
\begin{aligned}
& \text { Critical Value }=\frac{\text { Total } R P N}{\text { Number of Potential Hazards }} \\
& \text { Critical Value }=\frac{1313}{12} \\
& \text { Critical Value }=109
\end{aligned}
$$

Based on the order of RPN value results in Table 1, seven factors have the potential for danger in work above the critical Value, namely with the values of 193, 151, 149, 142, 139,131, and 115, respectively. Therefore, seven factors above the critical Value need to be resolved, and a solution is sought in advance to overcome the occurrence of accidents at work.

\section{Conclusions and Suggestions}

The results of this research are 12 potential hazards identified in the process of bread production in IKM Heppy Bakery using the FMEA method consisting of three variables, namely severity, occurrence, detection, and calculation of critical risk level (RPN) that is prioritized in repair. The result of data processing that has been obtained is the order of RPN values from the most considerable Value to the smallest Value. The enormous value obtained was 193, with the potential danger of overheating and dehydration operators. At the same time, the smallest value of 31 with the potential danger of gas leakage due to the location of gas is not appropriate. After obtaining the RPN value sequence, then determined the critical value of 12 potential hazard factors. The critical Value obtained is 109 , which indicates seven factors of potential hazards above the critical value. So, improvements need to be made in the form of solutions to overcome the seven potential hazards.

The solution that can be done to the potential dangers is to rearrange the layout of the machines so that in doing the work, the workers do not feel too overheated and the items placed to be more ergonomic so that workers do not easily experience fatigue when picking up goods. Workers should also pay attention to the condition of the floor of the production site so as not to slip during the production process.

Recommended suggestions for future research are: further research into identifying potential hazards can use methods other than the FMEA method. It should be necessary to make Standard Operating Procedures (SOP) related to the production work area at IKM Heppy Bakery. There need to be countermeasures to the repair solutions provided in IKM Heppy Bakery to increase productivity during bread production.

\section{References}

1. BBC NEWS.. Policy-by-policy: The coalition government's plans. BBC News Channel. (2010).

2. J. Doshi,., \& D. Desai,.. Application of failure mode \& effect analysis (FMEA) for continuous quality improvement - multiple case studies in automobile SMEs. International Journal for QualityResearch.0207. (2017).

3. P. Fithri, N. A. Riva, L Susanti, \& B. Yuliandra, Safety analysis at weaving department of PT. X Bogor 
using Failure Mode and Effect Analysis (FMEA) and Fault Tree Analysis (FTA). 2018 5th International Conference on Industrial Engineering and Applications, ICIEA 2018,382-385. (2018).

4. G. Halvani., M. Mohammadnia, M. Zare Mehrjardi, \& M. Keshtvarz. Risk assessment with intervention of gas pipelines repair using FMEA method and determination of safety performance monitoring: a case for a gas and oil operating company in 2012-13. Occupational Medicine.(2012).

5. G. B. HM. Analysis of Occupational Safety and Health in the Production Process Section Using the Failure Mode And Effect Analysis (FMEA) Method at PT Mitra Mandiri Perkasa. Jurnal IPTEK, 25(1). (2021).

6. T. Ishimar, H. Hirosato, T. Mori, N. Ito, K. Hiraoka, R. Minohara, S. Kajiki, M. Uehara, Y. Kobayashi, \& K. Mori. Systems and human resource development for occupational health in India: Effective occupational health management for Japanese enterprises with overseas branches. Sangyo Eiseigaku Zasshi = Journal of Occupational Health, 62(3).(2020).

7. M. Mangeli, A. Shahraki, \& F. H. Saljooghi, Improvement of risk assessment in the FMEA using nonlinear model, revised fuzzy TOPSIS, and support vector machine. International Journal of Industrial Ergonomics, 69. (2019).

8. Paciarotti, C., Mazzuto, G., \& D'Ettorre, D. A revised FMEA application to the quality control management. International Journal of Quality and Reliability Management, 31(7). (2014).

9. R. F. Ramadhan, E. Widowati, \& M. Mardiana. Failure Mode and Effect Analysis (FMEA) Application for Safety Risk Assessment Design of "X" Bakery. Unnes Journal of Public Health, 8(1). (2019). 